\title{
Density of eigenvalues of random normal matrices
}

\section{Journal Article}

Author(s):

Elbau, Peter; Felder, Giovanni (D)

Publication date:

2005-10

Permanent link:

https://doi.org/10.3929/ethz-b-000031810

\section{Rights / license:}

In Copyright - Non-Commercial Use Permitted

\section{Originally published in:}

Communications in Mathematical Physics 259(2), https://doi.org/10.1007/s00220-005-1372-z 


\title{
Density of Eigenvalues of Random Normal Matrices
}

\author{
Peter Elbau, Giovanni Felder \\ Department of Mathematics, ETH-Zentrum, 8092 Zurich, Switzerland. \\ E-mail: peter.elbau@math.ethz.ch; giovanni.felder@math.ethz.ch
}

Received: 27 September 2004 / Accepted: 7 February 2005

Published online: 14 June 2005 - () Springer-Verlag 2005

\begin{abstract}
The relation between random normal matrices and conformal mappings discovered by Wiegmann and Zabrodin is made rigorous by restricting normal matrices to have spectrum in a bounded set. It is shown that for a suitable class of potentials the asymptotic density of eigenvalues is uniform with support in the interior domain of a simple smooth curve.
\end{abstract}

\section{Introduction}

In recent work initiated by P. Wiegmann and A. Zabrodin [1-4], a connection between the normal matrix model and conformal mappings was discovered. In this model one considers random normal $N \times N$ complex matrices with probability measure

$$
P_{N}(M) \mathrm{d} M=Z_{N}^{-1} \exp \left\{-\left(N / t_{0}\right) \operatorname{tr}\left(M^{*} M-p(M)-p(M)^{*}\right)\right\} \mathrm{d} M,
$$

where $\mathrm{d} M$ is a natural measure on the variety of normal matrices and $Z_{N}$ is the normalization factor. The result is that, as $N \rightarrow \infty$, the density of eigenvalues is $1 / \pi t_{0}$ times the characteristic function of a bounded domain in the complex plane. This domain is characterized by the fact that its exterior harmonic moments are the coefficients $t_{j}$ of the polynomial $p$ appearing in the measure. Moreover, the Riemann mapping of the exterior of the unit disk onto the exterior of the domain obeys, as a function of the $t_{j}$, the equations of the integrable dispersionless Toda hierarchy.

These fascinating results remain however at the level of formal manipulations of undefined objects, as the integrals diverge, except in the simplest case of a polynomial $p$ of degree 2 , where the domain is bounded by an ellipse.

The purpose of this note is to give a setting in which the above statements make mathematical sense and to give a proof of these statements.

The problem of divergence of the integral over normal matrices is solved in a naive way, by restricting the integral to normal matrices whose eigenvalues lie in a compact 
domain $D$ of the complex plane. Then for small $t_{0}$ the results can be formulated in terms of polynomial curves, i.e., curves in the complex plane admitting a parametrization of the form $w \mapsto h(w)=r w+\sum_{j=0}^{n} a_{j} w^{-j},|w|=1$. For simple polynomial curves the problem of determining the curve out of its exterior harmonic moments $t_{j}=\frac{1}{2 \pi \mathrm{i}} \oint_{\gamma} \bar{z} z^{-j} \mathrm{~d} z$ and the area $\pi t_{0}$ of the interior domain (the interior domain is the bounded connected component of the complement of the curve) has a unique solution for small $t_{0}$, as we show in Sect. 5. Our main result is then:

Theorem 1.1. Let $D \subset \mathbb{C}$ be the closure of a bounded open set containing the origin. Let $p(z)=t_{2} z^{2}+\cdots+t_{n+1} z^{n+1}$ be a polynomial such that $|z|^{2}-p(z)-\overline{p(z)}$ has a non-degenerate absolute minimum in $D$ at $z=0$. Then there exists $a \delta>0$ so that for all $0<t_{0}<\delta$

(i) there exists a unique simple polynomial curve $\gamma$ with exterior harmonic moments $t_{1}=0, t_{2}, \ldots, t_{n+1}, 0,0, \ldots$ and area of interior domain $\pi t_{0}$;

(ii) the expectation value of the density of eigenvalues of random normal matrices with spectrum in $D$ and distribution (1) converges as $N \rightarrow \infty$ to a uniform distribution with support in the interior domain of $\gamma$.

The condition on $p$ implies the Hessian condition $\left|t_{2}\right|<\frac{1}{2}$ and it is fulfilled if the Hessian condition holds and $t_{3}, \ldots, t_{n}$ are sufficiently small. It then follows from results of $[5,1]$ that the curve $\gamma$ as a function of the $t_{j}$ in this range provides a solution of the integrable dispersionless $2 \mathrm{D}$ Toda hierarchy obeying the string equation.

The paper is organized as follows: the basic definitions of the random normal matrix model are recalled in Sect. 2. We then introduce the "equilibrium measure" as the unique solution of a variational problem in Sect. 3 and show in Sect. 4 that the density of eigenvalues converges to it. These are either known results or adaptations of results known for hermitian matrices to our case. In Sect. 5 we introduce the notion of polynomial curve and prove Theorem 5.3, which is a stronger form of part (i) of Theorem 1.1. In Sect. 6 we prove part (ii) of Theorem 1.1, see Theorem 6.1, and discuss our results in Sect. 7.

\section{Eigenvalues of Random Normal Matrices}

We consider the probability measure

$$
P_{N}(M) \mathrm{d} M=\frac{1}{Z_{N}} \mathrm{e}^{-N \operatorname{tr} V(M)} \mathrm{d} M, \quad Z_{N}=\int_{\mathcal{N}_{N}(D)} P_{N}(M) \mathrm{d} M,
$$

defined by a potential $V$, on the set

$$
\mathcal{N}_{N}(D)=\left\{M \in \operatorname{Mat}_{\mathbb{C}}(N) \mid\left[M, M^{*}\right]=0, \sigma(M) \subset D\right\}
$$

of normal $N \times N$ complex matrices with spectrum in some compact domain $D \subset \mathbb{C}$.

The measure $\mathrm{d} M$ is the Riemannian volume form on (the smooth part of) $\mathcal{N}_{N}(D)$ with respect to the metric induced from the standard metric on the vector space $\mathbb{C}^{N^{2}}$ of all $N \times N$ matrices. In a parametrization by eigenvalues and unitary matrices it is given by [6]

$$
\mathrm{d} M=\mathrm{d} U \prod_{1 \leq i<j \leq N}\left|z_{i}-z_{j}\right|^{2} \prod_{i=1}^{N} \mathrm{~d}^{2} z_{i}
$$


where $M=U \operatorname{diag}\left(\left(z_{i}\right)_{i=1}^{N}\right) U^{*}$ and $\mathrm{d} U$ denotes the normalized $\mathrm{U}(N)$ invariant measure on $\mathrm{U}(N) / \mathrm{U}(1)^{N}$.

This leads to the probability measure

$$
\begin{aligned}
P_{N}\left(\left(z_{i}\right)_{i=1}^{N}\right) \prod_{i=1}^{N} \mathrm{~d}^{2} z_{i} & =\frac{1}{Z_{N}} \mathrm{e}^{-N \sum_{i=1}^{N} V\left(z_{i}\right)} \prod_{1 \leq i<j \leq N}\left|z_{i}-z_{j}\right|^{2} \prod_{i=1}^{N} \mathrm{~d}^{2} z_{i}, \\
Z_{N} & =\int_{D^{N}} \mathrm{e}^{-N \sum_{i=1}^{N} V\left(z_{i}\right)} \prod_{1 \leq i<j \leq N}\left|z_{i}-z_{j}\right|^{2} \prod_{i=1}^{N} \mathrm{~d}^{2} z_{i}
\end{aligned}
$$

on the space of eigenvalues $z_{i} \in D, 1 \leq i \leq N$.

\section{The Equilibrium Measure}

We are interested in the behavior of the function $P_{N}(z)$ as $N \rightarrow \infty$. Because the probability that two eigenvalues are equal is always zero, we may consider $P_{N}$ as a function on the set $D_{0}^{N}=\left\{z \in D^{N} \mid z_{i} \neq z_{j} \forall i \neq j\right\}$.

Introducing the probability measure

$$
\delta_{z}(A)=\frac{1}{N} \sum_{i=1}^{N} \chi_{A}\left(z_{i}\right), \quad z=\left(z_{i}\right)_{i=1}^{N},
$$

on $D\left(\chi_{A}\right.$ shall denote the characteristic function of the set $\left.A\right)$, we write for $z \in D_{0}^{N}$,

$$
P_{N}(z)=\frac{1}{Z_{N}} \exp \left(-N^{2}\left(\int V(\zeta) \mathrm{d} \delta_{z}(\zeta)+\iint_{\zeta \neq \xi} \log |\zeta-\xi|^{-1} \mathrm{~d} \delta_{z}(\xi) \mathrm{d} \delta_{z}(\zeta)\right)\right) .
$$

Letting $N \rightarrow \infty$, only the infimum of the coefficient of $-N^{2}$,

$$
\begin{aligned}
I_{0} & :=\inf _{\mu \in \mathcal{M}(D)} I(\mu), \\
I(\mu) & :=\int V(z) \mathrm{d} \mu(z)+\iint_{z \neq \zeta} \log |z-\zeta|^{-1} \mathrm{~d} \mu(\zeta) \mathrm{d} \mu(z),
\end{aligned}
$$

will be relevant. Here $\mathcal{M}(D)$ denotes the set of all Borel probability measures on $D$ without point masses (a measure with point masses could only arise from measures $\delta_{z}$ with some $z_{i}=z_{j}$, but then $\left.P_{N}(z)=0\right)$. Therefore, we safely can neglect the restriction on the double integral.

The precise sense in which the infimum of $I$ controls the large $N$ behavior of $P_{N}$ will be discussed in the next section. Here we consider this reasoning only as a motivation for introducing the variational problem.

Because of $I\left(\frac{\chi_{D}}{|D|} \lambda\right)<\infty(\lambda$ the Lebesgue measure on $\mathbb{C}$ and $|D|=\lambda(D)$ the area of $D), I_{0}$ is finite.

Definition. An equilibrium measure for $V$ on $D \subset \mathbb{C}$ is a Borel probability measure $\mu$ on $D$ without point masses so that $I(\mu)=I_{0}$.

Theorem 3.1. Every continuous function $V$ on a compact subset $D$ has a unique equilibrium measure. 
In the remaining part of this section we prove this theorem, which is a known fact from potential theory, see e.g. [7], and give necessary and sufficient conditions for $\mu$ to be an equilibrium measure. The constructions are adapted from the corresponding results for Hermitian matrices, see [9].

3.1. Existence. To show the infimum is achieved, we choose a sequence $\left(\mu_{n}\right)_{n=1}^{\infty}$ in $\mathcal{M}(D)$ with $I\left(\mu_{n}\right) \rightarrow I_{0}$.

Lemma 3.2. The space of all Borel probability measures on D is sequentially compact.

Proof. By the theorem of Riesz-Markov each Borel measure $\mu$ on $D$ corresponds to exactly one positive, linear functional $\phi_{\mu} \in C(D)^{*}$ and by the theorem of Alaoglu, the closed unit-sphere in $C(D)^{*}$ is weak-*-compact. Therefore, for each sequence $\left(\mu_{n}\right)_{n=1}^{\infty}$ or Borel probability measures on $D$, the sequence $\left(\phi_{\mu_{n}}\right)_{n=1}^{\infty}$ contains a weak-*-convergent subsequence $\left(\phi_{\mu_{n(k)}}\right)_{k=1}^{\infty}$, i.e.

$$
\exists \phi \in C(D)^{*}: \phi_{\mu_{n(k)}}(f) \rightarrow \phi(f) \quad \forall f \in C(D) .
$$

Now we find a measure $\mu$ on $D$ with $\phi=\phi_{\mu}$. This measure fulfills

$$
\int f \mathrm{~d} \mu_{n(k)} \rightarrow \int f \mathrm{~d} \mu \quad(k \rightarrow \infty) \quad \forall f \in C(D),
$$

and hence is again a Borel probability measure.

Because of this lemma there exists a convergent subsequence $\left(\mu_{n(k)}\right)$ of $\left(\mu_{n}\right)$ and a Borel probability measure $\mu$ with $\mu_{n(k)} \rightarrow \mu$.

To prove that $I(\mu)=I_{0}$, we estimate with an arbitrary real constant $L$,

$$
\begin{aligned}
\lim _{k \rightarrow \infty} I\left(\mu_{n(k)}\right) & =\lim _{k \rightarrow \infty} \int V(z) \mathrm{d} \mu_{n(k)}(z)+\lim _{k \rightarrow \infty} \iint \log |z-\zeta|^{-1} \mathrm{~d} \mu_{n(k)}(\zeta) \mathrm{d} \mu_{n(k)}(z) \\
& \geq \int V(z) \mathrm{d} \mu(z)+\lim _{k \rightarrow \infty} \iint \min \left\{\log |z-\zeta|^{-1}, L\right\} \mathrm{d} \mu_{n(k)}(\zeta) \mathrm{d} \mu_{n(k)}(z) .
\end{aligned}
$$

Approximating uniformly the second integrand according to the theorem of StoneWeierstraß up to $\varepsilon>0$ with a polynomial in $z, \bar{z}, \zeta$ and $\bar{\zeta}$ and using Fubini's theorem, we get a lower bound for the limit:

$$
\lim _{k \rightarrow \infty} I\left(\mu_{n(k)}\right) \geq \int V(z) \mathrm{d} \mu(z)+\iint \min \left\{\log |z-\zeta|^{-1}, L\right\} \mathrm{d} \mu(\zeta) \mathrm{d} \mu(z)-2 \varepsilon .
$$

Letting first $\varepsilon \rightarrow 0$ and then $L \rightarrow \infty$ shows that $\mu$ has no point masses (otherwise the right-hand side would diverge) and $I_{0}=I(\mu)$.

3.2. Uniqueness. Next, we want to show that there is exactly one measure $\mu \in \mathcal{M}(D)$ with $I(\mu)=I_{0}$. So suppose $\tilde{\mu} \in \mathcal{M}(D)$ also fulfills $I(\tilde{\mu})=I_{0}$. Then we consider the family

$$
\mu_{t}=t \tilde{\mu}+(1-t) \mu=\mu+t(\tilde{\mu}-\mu), t \in[0,1],
$$

in $\mathcal{M}(D)$. 
Lemma 3.3. Let $\mu$ and $\tilde{\mu}$ be probability measures such that the function $\log |z-\zeta|^{-1}$ is integrable with respect to $\mu \otimes \mu$ and to $\tilde{\mu} \otimes \tilde{\mu}$. Then $\log |z-\zeta|^{-1}$ is also integrable with respect to $\mu \otimes \tilde{\mu}$.

Additionally, we have the inequality

$$
\iint \log |z-\zeta|^{-1} \mathrm{~d}(\tilde{\mu}-\mu)(\zeta) \mathrm{d}(\tilde{\mu}-\mu)(z) \geq 0,
$$

with equality if and only if $\mu=\tilde{\mu}$.

Proof. We start with the distributional identity

$$
\int \log |z|^{-1} \triangle \varphi(z) \mathrm{d}^{2} z=-2 \pi \varphi(0)
$$

for any Schwarz function $\varphi$. Introducing the Fourier transform $\hat{\varphi}$ of $\varphi$, we find

$$
\begin{aligned}
\int \log |z|^{-1} \triangle \varphi(z) \mathrm{d}^{2} z & =-\int \frac{1}{|k|^{2}}\left(|k|^{2} \hat{\varphi}(k)\right) \mathrm{d}^{2} k \\
& =\frac{1}{2 \pi} \int \frac{1}{|k|^{2}} \int \Delta \varphi(z)\left(\mathrm{e}^{\frac{\mathrm{i}}{2}(k z+\bar{k} \bar{z})}-f(k)\right) \mathrm{d}^{2} z \mathrm{~d}^{2} k \\
& =\frac{1}{2 \pi} \iint \frac{1}{|k|^{2}}\left(\mathrm{e}^{\frac{\mathrm{i}}{2}(k z+\bar{k} \bar{z})}-f(k)\right) \mathrm{d}^{2} k \Delta \varphi(z) \mathrm{d}^{2} z,
\end{aligned}
$$

where $f$ denotes a real, continuous function which is one in the vicinity of zero and becomes zero at infinity. So we have for all $z \in \mathbb{C} \backslash\{0\}$ the equation

$$
\log |z|^{-1}=\frac{1}{2 \pi} \int \frac{1}{|k|^{2}}\left(\mathrm{e}^{\frac{\mathrm{i}}{2}(k z+\bar{k} \bar{z})}-f(k)\right) \mathrm{d}^{2} k+C(f)
$$

with some real constant $C(f)$.

So, with Tonelli's theorem, we see that

$$
\begin{aligned}
& \iint \log |z-\zeta|^{-1} \mathrm{~d}(\tilde{\mu}-\mu)(\zeta) \mathrm{d}(\tilde{\mu}-\mu)(z) \\
& \quad=\frac{1}{2 \pi} \int \frac{1}{|k|^{2}}\left|\int \mathrm{e}^{\frac{\mathrm{i}}{2}(k z+\bar{k} \bar{z})} \mathrm{d}(\tilde{\mu}-\mu)(z)\right|^{2} \mathrm{~d}^{2} k
\end{aligned}
$$

is non-negative and finite, which immediately implies the integrability of $\log |z-w|^{-1}$ with respect to $\mu \otimes \tilde{\mu}$. To achieve equality in (2), we need

$$
\int \mathrm{e}^{\frac{\mathrm{i}}{2}(k z+\bar{k} \bar{z})} \mathrm{d} \mu(z)=\int \mathrm{e}^{\frac{\mathrm{i}}{2}(k z+\bar{k} \bar{z})} \mathrm{d} \tilde{\mu}(z)
$$

for all $k \in \mathbb{C}$, which reads $\mu=\tilde{\mu}$.

So we can expand $I\left(\mu_{t}\right)$ and obtain

$$
\begin{aligned}
I\left(\mu_{t}\right)= & I(\mu)+t \int\left(V(z)+2 \int \log |z-\zeta|^{-1} \mathrm{~d} \mu(\zeta)\right) \mathrm{d}(\tilde{\mu}-\mu)(z) \\
& +t^{2} \iint \log |z-\zeta|^{-1} \mathrm{~d}(\tilde{\mu}-\mu)(\zeta) \mathrm{d}(\tilde{\mu}-\mu)(z) .
\end{aligned}
$$


Lemma 3.3 now states that the coefficient of $t^{2}$ is non-negative and so the function $t \mapsto I\left(\mu_{t}\right)$ is convex on $[0,1]$. In particular,

$$
I\left(\mu_{t}\right) \leq t I(\tilde{\mu})+(1-t) I(\mu)=I_{0},
$$

which implies $I\left(\mu_{t}\right) \equiv I_{0}$. This requires the last summand in (3) to vanish and so, again with Lemma 3.3, we see that $\mu=\tilde{\mu}$.

3.3. A variational form. To determine if a given measure $\mu$ is the equilibrium measure for the potential $V$ on the domain $D$, we may check a variational principle.

Proposition 3.4. The probability measure $\mu$ is the equilibrium measure for the potential $V$ on the domain $D$ if and only if the function

$$
E(z)=V(z)+2 \int \log |z-\zeta|^{-1} \mathrm{~d} \mu(\zeta)
$$

fulfills the relation

$$
\int E(z) \mathrm{d} \tilde{\mu}(z) \geq \int E(z) \mathrm{d} \mu(z)=: E_{0} \quad \text { for all } \tilde{\mu} \in \mathcal{M}(D) .
$$

Additionally, we have the property

$$
E(z) \equiv E_{0} \quad \mu \text {-almost everywhere. }
$$

Proof. Let us first assume $\mu$ is the equilibrium measure. Then, for an arbitrary measure $\tilde{\mu} \in \mathcal{M}(D)$, the condition

$$
\left.\frac{\mathrm{d}}{\mathrm{d} t} I\left(\mu_{t}\right)\right|_{t=0} \geq 0, \quad \mu_{t}=t \tilde{\mu}+(1-t) \mu, \quad t \in[0,1],
$$

has to hold. This means

$$
\int\left(V(z)+2 \int \log |z-\zeta|^{-1} \mathrm{~d} \mu(\zeta)\right) \mathrm{d}(\tilde{\mu}-\mu)(z) \geq 0,
$$

which immediately implies Eq. (5).

Assume on the other side $\mu$ fulfills condition (5). Then we obtain with the equilibrium measure $\mu_{0}$,

$$
\begin{aligned}
I\left(\mu_{0}\right)=I(\mu)+ & \int\left(V(z)+2 \int \log |z-\zeta|^{-1} \mathrm{~d} \mu(\zeta)\right) \mathrm{d}\left(\mu_{0}-\mu\right)(z) \\
& +\iint \log |z-\zeta|^{-1} \mathrm{~d}\left(\mu_{0}-\mu\right)(\zeta) \mathrm{d}\left(\mu_{0}-\mu\right)(z) \\
\geq I(\mu), &
\end{aligned}
$$

and so $\mu=\mu_{0}$.

To show the additional statement, we consider for the probability measure $\mu$ the set

$$
B=\left\{z \in D \mid E(z)<E_{0}\right\} .
$$

If $\mu(B)>0$, the variational principle (5) for the measure $\tilde{\mu}=\frac{\chi_{B}}{\mu(B)} \mu$ would yield

$$
E_{0} \leq \int E(z) \frac{\chi_{B}(z)}{\mu(B)} \mathrm{d} \mu(z)<E_{0},
$$

and so $\mu(B)$ has to vanish. Thus, we get condition (6).

Instead of verifying condition (5), we prefer to use the following statement: 
Corollary 3.5. If for a measure $\mu \in \mathcal{M}(D)$ the function $E$, defined by Eq. (4), fulfills, for some real constant $E_{0}, E(z) \equiv E_{0}$ on the support of $\mu$ and $E(z) \geq E_{0}$ everywhere, then $\mu$ is the equilibrium measure.

\section{The Eigenvalue Density}

Definition. The k-point correlation function $R^{(k)}$ is given by

$$
R_{N}^{(k)}\left(\left(z_{i}\right)_{i=1}^{k}\right)=\frac{N !}{(N-k) !} \int_{D^{N-k}} P_{N}\left(\left(z_{i}\right)_{i=1}^{N}\right) \prod_{i=k+1}^{N} \mathrm{~d}^{2} z_{i} .
$$

So, the one-point correlation function is up to normalization nothing but the density of the eigenvalues.

As indicated in the previous section, all the correlation functions can be calculated in the limit $N \rightarrow \infty$ out of the equilibrium measure $\mu$, as in the case of hermitian matrix models, see $[8,9]$.

Theorem 4.1. For all $\phi \in C\left(D^{k}\right)$ we have the equality

$$
\lim _{N \rightarrow \infty} \int_{D^{k}} \frac{1}{N^{k}} \phi\left(\left(z_{i}\right)_{i=1}^{k}\right) R_{N}^{(k)}\left(\left(z_{i}\right)_{i=1}^{k}\right) \prod_{i=1}^{k} \mathrm{~d}^{2} z_{i}=\int \phi\left(\left(z_{i}\right)_{i=1}^{k}\right) \prod_{i=1}^{k} \mathrm{~d} \mu\left(z_{i}\right) .
$$

I.e. the measure $\frac{1}{N^{k}} R_{N}^{(k)}\left(\left(z_{i}\right)_{i=1}^{k}\right) \prod_{i=1}^{k} \mathrm{~d}^{2} z_{i}$ on $D^{k}$ converges weakly to $\prod_{i=1}^{k} \mathrm{~d} \mu\left(z_{i}\right)$.

Proof. Substituting in the left-hand-side of Eq. (7) the definition of the correlation functions and turning our attention to the highest order in $N$, we obtain (because $P_{N}$ is invariant under the symmetric group)

$$
\begin{aligned}
\left\langle\phi, \frac{1}{N^{k}} R_{N}^{(k)}\right\rangle & =\frac{1}{N^{k}} \int_{D^{k}} \phi\left(\left(z_{i}\right)_{i=1}^{k}\right) R_{N}^{(k)}\left(\left(z_{i}\right)_{i=1}^{k}\right) \prod_{i=1}^{k} \mathrm{~d}^{2} z_{i} \\
& =\frac{1}{N^{k}} \sum_{i_{1}, \ldots, i_{k}=1}^{N} \int_{D^{k}} \phi\left(\left(z_{i_{j}}\right)_{j=1}^{k}\right) P_{N}\left(\left(z_{i}\right)_{i=1}^{N}\right) \prod_{i=1}^{N} \mathrm{~d}^{2} z_{i}+o(1) .
\end{aligned}
$$

Since for large values of $N$ the probability distribution localizes at values $z \in D_{0}^{N}$ with $I\left(\delta_{z}\right) \approx I_{0}$, let us consider the sets

$$
A_{N, \eta}=\left\{z \in D_{0}^{N} \mid I\left(\delta_{z}\right) \leq I_{0}+\eta\right\}, \quad \eta>0 .
$$

Lemma 4.2. The probability $P_{N}\left(D^{N} \backslash A_{N, \eta}\right)$ drops for $N \rightarrow \infty$ exponentially to zero.

Proof. For an absolutely continuous ${ }^{1}$ equilibrium measure $\mathrm{d} \mu(z)=\psi(z) \mathrm{d}^{2} z$, we get with Jensen's theorem and $\int I\left(\delta_{z}\right) \prod \mathrm{d} \mu\left(z_{i}\right)=I_{0}+o(1)$,

$$
Z_{N} \geq \int_{\left\{z \in D^{N} \mid \psi\left(z_{i}\right) \neq 0 \forall i\right\}} \mathrm{e}^{-N^{2} I\left(\delta_{z}\right)-\sum_{i=1}^{N} \log \psi\left(z_{i}\right)} \prod_{i=1}^{N} \mathrm{~d} \mu\left(z_{i}\right) \geq \mathrm{e}^{-N^{2} I_{0}+o\left(N^{2}\right)},
$$

1 This case suffices for our needs, but the restriction is in fact not necessary. Indeed, we could perform an analogous argument for the measures $\mathrm{d} \mu_{\varepsilon}(z)=\psi_{\varepsilon}(z) \mathrm{d}^{2} z, \psi_{\varepsilon}(z)=\frac{1}{\pi \varepsilon^{2}} \int_{B_{\varepsilon}(z)} \mathrm{d} \mu$. In the limit $\varepsilon \rightarrow 0$, where $I\left(\mu_{\varepsilon}\right) \rightarrow I_{0}$, this would yield the desired statement. 
and therefore,

$$
P_{N}\left(D^{N} \backslash A_{N, \eta}\right) \leq \int_{D^{N}} \mathrm{e}^{N^{2} I_{0}+o\left(N^{2}\right)-N^{2}\left(I_{0}+\eta\right)} \prod_{i=1}^{N} \mathrm{~d}^{2} z_{i}=o\left(\mathrm{e}^{-N^{2} \eta / 2}\right) .
$$

Let the continuous function $\frac{1}{N^{k}} \sum \phi\left(\left(z_{i j}\right)_{j=1}^{k}\right)$ take its maximum on the compact set $A_{N, \eta}$ at $\zeta$, and set $\nu_{N, \eta}=\delta_{\zeta}$. Then,

$$
\left\langle\phi, \frac{1}{N^{k}} R_{N}^{(k)}\right\rangle \leq \frac{1}{N^{k}} \sum_{i_{1}, \ldots, i_{k}=1}^{N} \phi\left(\left(\zeta_{i_{j}}\right)_{j=1}^{k}\right)=\int \phi\left(\left(z_{i}\right)_{i=1}^{k}\right) \prod_{i=1}^{k} \mathrm{~d} v_{N, \eta}\left(z_{i}\right) .
$$

Because of Lemma 3.2, we find a convergent subsequence $v_{N(n), \eta} \rightarrow v_{\eta}(n \rightarrow \infty)$ with

$$
\varlimsup_{N \rightarrow \infty}\left\langle\phi, \frac{1}{N^{k}} R_{N}^{(k)}\right\rangle \leq \int \phi\left(\left(z_{i}\right)_{i=1}^{k}\right) \prod_{i=1}^{k} \mathrm{~d} v_{\eta}\left(z_{i}\right) .
$$

Lemma 4.3. We have $v_{\eta} \in \mathcal{M}(D)$ and, in the limit $\eta \rightarrow 0, I\left(v_{\eta}\right) \rightarrow I_{0}$.

Proof. Using $\zeta \in A_{N(n), \eta}$, we obtain with the cut-off $L \in \mathbb{R}$ :

$$
\begin{aligned}
I_{0}+\eta \geq & \frac{1}{N(n)} \sum_{i=1}^{N(n)} V\left(\zeta_{i}\right)+\frac{1}{N(n)^{2}} \sum_{1 \leq i \neq j \leq N(n)} \min \left\{\log \left|\zeta_{i}-\zeta_{j}\right|^{-1}, L\right\} \\
= & \int V(z) \mathrm{d} v_{N(n), \eta}(z) \\
& +\iint \min \left\{\log |z-\zeta|^{-1}, L\right\} \mathrm{d} v_{N(n), \eta}(\zeta) \mathrm{d} v_{N(n), \eta}(z)-\frac{L}{N(n)}
\end{aligned}
$$

Sending first $n$ and then $L$ to infinity brings us to $v_{\eta} \in \mathcal{M}(D)$ and therefore $I_{0} \leq I\left(v_{\eta}\right) \leq I_{0}+\eta$.

So, letting $\eta \rightarrow 0$, a subsequence of $v_{\eta}$ converges to the equilibrium measure $\mu$, and thus,

$$
\varlimsup_{N \rightarrow \infty}\left\langle\phi, \frac{1}{N^{k}} R_{N}^{(k)}\right\rangle \leq \int \phi\left(\left(z_{i}\right)_{i=1}^{k}\right) \prod_{i=1}^{k} \mathrm{~d} \mu\left(z_{i}\right) .
$$

Arguing in the same way for the limes inferior concludes the proof.

\section{Polynomial Curves}

Definition. A polynomial curve of degree $n$ is a smooth simple closed curve in the complex plane with a parametrization $h: S^{1} \subset \mathbb{C} \rightarrow \mathbb{C}$ of the form

$$
h(w)=r w+a_{0}+a_{1} w^{-1}+\cdots+a_{n} w^{-n}, \quad|w|=1,
$$

with $r>0$ and $a_{n} \neq 0$. The standard (counterclockwise) orientation of the circle induces an orientation on the curve. We say that a polynomial curve is positively oriented if this orientation is counterclockwise, i.e., if the tangent vector to the curve makes one full turn in the counterclockwise direction as we go around the unit circle. 
Proposition 5.1. Let $\gamma$ be a positively oriented polynomial curve with parametrization $h$ of the form (8). Then $h$, viewed as a homolorphic map on $\mathbb{C}^{\times}$, restricts to a biholomorphic map from the exterior of the unit disk onto the exterior of $\gamma$.

Proof. We have to show that $h^{\prime}(w) \neq 0$ for all $w$ in the complement of the unit disk. Let $t$ denote the tangent vector map $w \mapsto t(w)=h^{\prime}(w) \mathrm{i} w=\mathrm{i}\left(r w-\sum j a_{j} w^{-j}\right)$. Since $\gamma$ is a simple closed curve, the map $w \mapsto t(w) /|t(w)|$ is a map of degree 1 from the unit circle to itself. Therefore we have

$$
\begin{aligned}
1 & =\frac{1}{2 \pi} \oint_{|w|=1} \mathrm{~d} \arg (t(w)) \\
& =\frac{1}{2 \pi \mathrm{i}} \oint_{|w|=1} \frac{t^{\prime}(w)}{t(w)} \mathrm{d} w \\
& =N+\frac{1}{2 \pi \mathrm{i}} \oint_{|w|=R} \frac{t^{\prime}(w)}{t(w)} \mathrm{d} w .
\end{aligned}
$$

Here $N \geq 0$ denotes the number of zeros of $t(w)$, counted with multiplicity, in the complement of the unit disk and $R$ is so large that it contains them all. The latter integral is 1 as can be seen by sending $R$ to infinity. Thus $N=0$, and $h^{\prime}$ has no zeros in the complement of the unit disk.

A simple consequence of this proposition is that a polynomial curve is uniquely parametrized by a map of the form (8) with $r>0$. Indeed, any other conformal mapping of the complement differs by an automorphism of the complement of the unit disk. But non-trivial automorphisms are given by fractional linear transformations which do not preserve the conditions.

From now on, we will only consider polynomial curves encircling the origin, i.e., such that the origin is contained in their interior domain. This can always be achieved by a translation, i.e., a shift of $a_{0}$.

Definition. The harmonic moments $\left(t_{j}\right)_{j=1}^{\infty}$ of the exterior domain $D_{-}$of a polynomial curve (or more generally of an analytic curve) encircling the origin are defined by

$$
t_{j}=-\frac{1}{\pi j} \int_{D_{-}} z^{-j} \mathrm{~d}^{2} z=\frac{1}{2 \pi \mathrm{i} j} \oint_{\gamma} \bar{z} z^{-j} \mathrm{~d} z,
$$

where only the right integral should be taken as a definition for $j \leq 2$.

Proposition 5.2. Let $\gamma$ be a positively oriented polynomial curve of degree $n$ encircling the origin.

(i) The exterior harmonic moments $t_{j}$ of $\gamma$ vanish for all $j>n+1$.

(ii) There exist universal polynomials $P_{j, k} \in \mathbb{Z}\left[r, a_{0}, \ldots, a_{k-j}\right], 1 \leq j \leq k$, so that for $j=1, \ldots, n+1$,

$$
j t_{j}=\bar{a}_{j-1} r^{-j+1}+\sum_{k=j}^{n} \bar{a}_{k} r^{-k} P_{j, k}\left(r, a_{0}, \ldots, a_{k-j}\right) .
$$

Moreover, $P_{j, k}$ is a homogeneous polynomial of degree $k-j+1$ and it is also weighted homogeneous of degree $k-j+1$ for the assignment $\operatorname{deg}\left(a_{j}\right)=j+1$, $\operatorname{deg}(r)=0$. 
(iii) The area of the domain enclosed by $\gamma$ is $\pi t_{0}$, where

$$
t_{0}=r^{2}-\sum_{j=1}^{n} j\left|a_{j}\right|^{2} .
$$

Proof. Since $\gamma$ encircles the origin, $h(w)$ never vanishes for $|w| \geq 1$. Hence the contour in the formula for $t_{j}$ may be computed by taking residues at infinity. For $j \geq 1$,

$$
\begin{aligned}
j t_{j} & =\frac{1}{2 \pi \mathrm{i}} \oint_{|w|=1} \bar{h}\left(w^{-1}\right) h^{\prime}(w) h(w)^{-j} \mathrm{~d} w \\
& =r^{-j} \sum_{k=0}^{n} \frac{\bar{a}_{k}}{2 \pi \mathrm{i}} \oint w^{k-j}\left(r-\sum_{l=1}^{n} l a_{l} w^{-l-1}\right)\left(1+\sum_{l=0}^{n} a_{l} w^{-l-1} / r\right)^{-j} \mathrm{~d} w .
\end{aligned}
$$

The integrals in this sum vanish if $k \leq j-2$. The formula for $t_{j}$ in terms of $a_{k}, r$ is obtained by expanding the geometric series and picking the coefficient of $w^{-1}$ in the integrand. This proves (i) and the first part of (ii). The homogeneity property is clear. The weighted homogeneity follows by rescaling $w$ in the integral. The same formula can be used to compute $t_{0}$, but the first term $r w^{-1}$ in $\bar{h}\left(w^{-1}\right)$, which does not contribute to the integral and was omitted for $j \geq 1$, must be added here.

Examples. The terms in $t_{j}$ involving polynomials $P_{j, k}$ with $k \leq 3$ are

$$
\begin{aligned}
t_{1} & =\bar{a}_{0}-r^{-1} \bar{a}_{1} a_{0}-r^{-2} \bar{a}_{2}\left(2 a_{1} r-a_{0}^{2}\right)+r^{-3} \bar{a}_{3}\left(3 a_{0} a_{1} r-3 a_{2} r^{2}-a_{0}{ }^{3}\right)+\cdots, \\
2 t_{2} & =r^{-1} \bar{a}_{1}-2 r^{-2} \bar{a}_{2} a_{0}-3 r^{-3} \bar{a}_{3}\left(a_{1} r-a_{0}^{2}\right)+\cdots, \\
3 t_{3} & =r^{-2} \bar{a}_{2}-3 r^{-3} \bar{a}_{3} a_{0}+\cdots .
\end{aligned}
$$

Theorem 5.3. Let $t_{2}, \ldots, t_{n+1}$ be complex numbers so that $\left|t_{2}\right|<1 / 2$. Then there exists an $A_{0}=A_{0}\left(t_{2}, \ldots, t_{n+1}\right)>0$ so that for all $A, t_{1}$ with $0<A<A_{0}$ and $\left|t_{1}\right|^{2}<$ $A\left(1 / 2-\left|t_{2}\right|\right)$, there exists a unique positively oriented polynomial curve of degree $\leq n$ encircling the origin, with area A and exterior harmonic moments $t_{1}, \ldots, t_{n+1}, 0,0, \ldots$

Proof. The idea is to invert the map $\left(r, a_{0}, \ldots, a_{n}\right) \mapsto\left(t_{0}, \ldots, t_{n+1}\right)$ for small $r$ and $a_{0}$. Set $\alpha_{j}=r^{-j} a_{j}, \rho=r^{2}$ and consider instead the polynomial map

$$
F:\left(\rho, \alpha_{0}, \ldots, \alpha_{n}\right) \rightarrow\left(t_{0}, \ldots, t_{n+1}\right),
$$

as a map from $\mathbb{R} \times \mathbb{C}^{n+1}$ to itself. The first claim is that this map has a smooth inverse in some neighborhood of any point $t \in \mathbb{R} \times \mathbb{C}^{n+1}$ such that $t_{0}=t_{1}=0$ and $\left|t_{2}\right| \neq 1 / 2$. By Prop. 5.2, this map is given by

$$
\begin{aligned}
t_{0} & =\rho-\sum_{j=1}^{n} \rho^{j} j\left|\alpha_{j}\right|^{2}, \\
j t_{j} & =\bar{\alpha}_{j-1}+\sum_{k=j}^{n} \bar{\alpha}_{k} P_{j, k}\left(r, \alpha_{0}, r \alpha_{1}, \ldots, r^{k-j} \alpha_{k-j}\right) \\
& =\bar{\alpha}_{j-1}+\sum_{k=j}^{n} \bar{\alpha}_{k} P_{j, k}\left(\rho, \alpha_{0}, \alpha_{1}, \ldots, \alpha_{k-j}\right) .
\end{aligned}
$$


From the contour integral representation of the harmonic moments we get the integral

$$
\begin{aligned}
& P_{j, k}\left(\rho, \alpha_{0}, \ldots, \alpha_{k-j}\right) \\
& \quad=\frac{1}{2 \pi i} \oint_{|w|=R} w^{k-j}\left(1-\sum_{l=1}^{n} l \alpha_{l} \rho^{l} w^{-l-1}\right)\left(1+\sum_{l=0}^{n} \alpha_{l} \rho^{l} w^{-l-1}\right)^{-j} \mathrm{~d} w
\end{aligned}
$$

for any sufficiently large $R$. By computing the residue at infinity, we can calculate $P_{j, k}$ and thus $t_{j}$ up to terms of at least second order in $\alpha_{0}, \rho$,

$$
\begin{aligned}
t_{0} & =\rho\left(1-\left|\alpha_{1}\right|^{2}\right)+\cdots, \\
j t_{j} & =\bar{\alpha}_{j-1}-j \bar{\alpha}_{j} \alpha_{0}-(j+1) \rho \bar{\alpha}_{j+1} \alpha_{1}+\cdots, \quad j \geq 1 .
\end{aligned}
$$

Hence $F\left(0,0,2 \bar{t}_{2}, \ldots,(n+1) \bar{t}_{n+1}\right)=\left(0,0, t_{2}, \ldots, t_{n+1}\right)$ and the tangent map at this point sends $\left(\dot{\rho}, \dot{\alpha}_{0}, \ldots, \dot{\alpha}_{n}\right)$ to $\left(\dot{t}_{0}, \ldots, \dot{t}_{n}\right)$ with

$$
\begin{aligned}
\dot{t}_{0} & =\left(1-4\left|t_{2}\right|^{2}\right) \dot{\rho} \\
j \dot{t}_{j} & =\overline{\dot{\alpha}}_{j-1}-j(j+1) t_{j+1} \dot{\alpha}_{0}-2 j(j+1)(j+2) t_{j+2} t_{2} \dot{\rho}, \quad j \geq 1 .
\end{aligned}
$$

The tangent map is invertible if $\left|t_{2}\right| \neq 1 / 2$. By the inverse function theorem, $F$ has a smooth inverse on some neighborhood of $\left(0,0, t_{2}, \ldots, t_{n+1}\right)$. If $\left|t_{2}\right|<1 / 2, F$ preserves the positivity of the first coordinate.

In terms of the original variables, this means that given any $t=\left(t_{0}, t_{1}, t_{2}, \ldots, t_{n+1}\right)$ with small $t_{0}>0, t_{1}$ and such that $\left|t_{2}\right| \neq 1 / 2$, there is a curve $w \mapsto h(w)$ with $h(w)=r w+\alpha_{0}+r \alpha_{1} w^{-1}+\cdots+r^{n} \alpha_{n} w^{-n}$ and $\alpha_{j} \simeq(j+1) \bar{t}_{j+1}$. It remains to show that if $r>0$ is small enough, $h$ parametrizes a positively oriented simple closed curve containing the origin. We first show that $h$ is an immersion. Since $h^{\prime}(w)=$ $r-r \alpha_{1} w^{-2}+O\left(r^{2}\right)$ and $\lim _{r \rightarrow 0} \alpha_{1}=2 \bar{t}_{2}$, we see that as long as $\left|t_{2}\right| \neq 1 / 2, h^{\prime}(w)$ does not vanish on the unit circle. Similarly, we show that $h: S^{1} \rightarrow \mathbb{C}$ is injective: we have

$$
\begin{aligned}
\left|h(w)-h\left(w^{\prime}\right)\right|^{2} & =r\left|w-w^{\prime}+2 \bar{t}_{2}\left(w^{-1}-w^{\prime}-1\right)\right|+O\left(r^{2}\right) \\
& =r\left|w-w^{\prime}+2 \bar{t}_{2}\left(\bar{w}-\bar{w}^{\prime}\right)\right|+O\left(r^{2}\right) .
\end{aligned}
$$

But the expression in the absolute value can only vanish for $w \neq w^{\prime}$ if $\left|2 \bar{t}_{2}\right|=1$ which is excluded by the hypothesis. Moreover $h(w)=r w+\bar{t}_{1}+2 r \bar{t}_{2} w^{-1}+O\left(r^{2}\right)$. Therefore $h$ parametrizes a perturbation of an ellipse centered at $\bar{t}_{1}$. The condition on $t_{1}$ is a sufficient condition for this ellipse to contain the origin.

Example. Let $k \geq 3$ and let us consider curves with $t_{j}=0$ for all $j \neq k$. Then $a_{j}=0$ for all $j \neq k-1$, so that $h(w)=r w+a_{k-1} w^{-k+1}$. The relation between $\left(t_{0}, t_{k}\right)$ and $\left(r, a_{k-1}\right)$ is $t_{0}=r^{2}-(k-1)\left|a_{k-1}\right|^{2}, k t_{k}=\bar{a}_{k-1} r^{-k+1}$. This map is a diffeomorphism from the region $0<r<(k-1)\left|a_{k-1}\right|$, which is the condition for $h(w)$ to be an embedding of the unit circle, onto the region

$$
0<t_{0}<\left(k(k-1)\left|t_{k}\right|\right)^{-2 /(k-2)}(k-2) /(k-1) .
$$

As $t_{0}$ approaches the upper bound for given $t_{k}$, the curve develops cusp singularities. 


\section{The Equilibrium Measure for a Polynomial Curve}

In this section we evaluate the equilibrium measure corresponding to potentials

$$
V(z)=\frac{1}{t_{0}}\left(|z|^{2}-2 \operatorname{Re} \sum_{k=1}^{n+1} t_{k} z^{k}\right)
$$

We anticipate the result:

Theorem 6.1. For any set $\left(t_{k}\right)_{k=1}^{\infty} \subset \mathbb{C}$ with $t_{1}=0,\left|t_{2}\right|<\frac{1}{2}$ and $t_{k}=0$ for $k>n+1$ and any compact domain $D \subset \mathbb{C}$ containing the origin as an interior point and such that $t_{0} V, V$ given by Eq. (10), is positive on $D \backslash\{0\}$, there exists a $\delta>0$ so that for all $0<t_{0}<\delta$ the equilibrium measure $\mu$ for $V$ on $D$ is given by

$$
\mu=\frac{1}{\pi t_{0}} \chi_{D_{+}} \lambda
$$

where $D_{+}$denotes the interior domain of the polynomial curve $\gamma$ defined by the harmonic moments $\left(t_{k}\right)_{k=0}^{\infty}$, and $\lambda$ is the Lebesgue measure on $\mathbb{C}$.

The rest of the section is dedicated to the proof of this theorem.

6.1. The Schwarz reflection. We first need the notion of a reflection on an analytic curve (see e.g. [10]).

Definition. The Schwarz function of an analytic curve $\gamma$ is defined as the analytic continuation (in a neighbourhood of the curve) of the function $S(z)=\bar{z}$ on $\gamma$. The Schwarz reflection $\rho$ for the analytic curve in this domain is the anti-holomorphic map $\rho(z)=\overline{S(z)}$.

Definition. Under the critical radius $R$ of the polynomial curve defined by the parametrization $h$ we understand the value

$$
R=\max \left\{|w| \mid h^{\prime}(w)=0, w \in \mathbb{C}\right\},
$$

which, by definition of the map $h$, is less than 1.

Lemma 6.2. Let $\gamma$ be a polynomial curve parametrized by $h$ and $R$ its critical radius. Then the Schwarz function $S$ and the Schwarz reflection $\rho$ of the curve $\gamma$ restricted to $h\left(B_{1 / R} \backslash \bar{B}_{R}\right)$, where $B_{R}$ denotes the open disk with radius $R$ around zero, are biholomorphic respectively anti-biholomorphic maps. They are given by

$$
S(z)=\bar{h}\left(\frac{1}{h^{-1}(z)}\right) \quad \text { and } \quad \rho(z)=h\left(\frac{1}{\bar{h}^{-1}(\bar{z})}\right) \text {. }
$$

Therefore, $\rho$ maps $\gamma$ identically on itself, $h\left(B_{1} \backslash \bar{B}_{R}\right)$ to $h\left(B_{1 / R} \backslash \bar{B}_{1}\right)$ and vice versa. Also, we have $\rho^{2}=\mathrm{id}$. 
Proof. By definition of the Schwarz function, in a neighborhood of $|w|=1$,

$$
S(h(w))=\bar{h}\left(w^{-1}\right) .
$$

Because for $w \in \mathbb{C} \backslash \bar{B}_{R}$ the function $h$ is biholomorphic, we may write

$$
S(z)=\bar{h}\left(\frac{1}{h^{-1}(z)}\right), \quad \rho(z)=\overline{S(z)}=h\left(\frac{1}{\bar{h}^{-1}(\bar{z})}\right) .
$$

Taking the derivatives, we find that they do not vanish for $R<\left|h^{-1}(z)\right|<\frac{1}{R}$.

Lemma 6.3. In the interior domain $D_{+}$of the polynomial curve defined by the parameters $t_{k}$ from (10) as its harmonic moments, the function

$$
E(z)=V(z)+\frac{2}{\pi t_{0}} \int_{D_{+}} \log \left|\frac{z}{\zeta}-1\right|^{-1} \mathrm{~d}^{2} \zeta
$$

is equal to zero and in the exterior domain $D \backslash D_{+}$, its gradient reads

$$
\partial_{\bar{z}} E(z)=\frac{1}{t_{0}}(z-\rho(z)) .
$$

Proof. To verify the first statement, we use Green's theorem and obtain

$$
\frac{2}{\pi} \int_{D_{+}} \log |z-\zeta|^{-1} \mathrm{~d}^{2} \zeta=-|z|^{2}+\operatorname{Re} \frac{1}{2 \pi \mathrm{i}} \oint_{\gamma}\left(\log |z-\zeta|^{-1} \bar{\zeta}+\frac{|\zeta|^{2}}{\zeta-z}\right) \mathrm{d} \zeta .
$$

Integrating by parts of the second integrand yields immediately

$$
\frac{2}{\pi} \int_{D_{+}} \log |z-\zeta|^{-1} \mathrm{~d}^{2} \zeta=-|z|^{2}-2 \operatorname{Re} \frac{1}{2 \pi \mathrm{i}} \oint_{\gamma} \log (\zeta-z) \bar{\zeta} \mathrm{d} \zeta
$$

and expanding the logarithm around $z=0$ leads us to $E(z)=0$ in $D_{+}$.

For the proof of the second part we write $S=S_{\mathrm{i}}+S_{\mathrm{e}}$, where $S_{\mathrm{i}}$ is analytic in $D_{+}$and $S_{\mathrm{e}}$ in the complement $D \backslash D_{+}$. For the exterior function $S_{\mathrm{e}}$ one finds with the Cauchy integral and Stokes formula:

$$
S_{\mathrm{e}}(z)=-\frac{1}{2 \pi \mathrm{i}} \oint_{\gamma} \frac{\bar{\zeta}-S_{\mathrm{i}}(\zeta)}{\zeta-z} \mathrm{~d} \zeta=\frac{1}{\pi} \int_{D_{+}} \frac{1}{z-\zeta} \mathrm{d}^{2} \zeta, \quad z \in D \backslash D_{+} .
$$

Because we know $E$ to be constant on $D_{+}$,

$$
0=\partial_{z} E(z)=\frac{1}{t_{0}}\left(\bar{z}-\sum_{k=1}^{n+1} k t_{k} z^{k-1}-\frac{1}{\pi} \int_{D_{+}} \frac{1}{z-\zeta} \mathrm{d}^{2} \zeta\right)
$$

for all $z \in D_{+}$. So on im $\gamma$, and by analytic continuation in the entire domain where $S$ is holomorphic (which includes the exterior domain $D \backslash D_{+}$), we have $S_{\mathrm{i}}(z)=\sum_{k=1}^{n+1} k t_{k} z^{k-1}$.

Therefore, for all $z \in D \backslash D_{+}$,

$$
\partial_{\bar{z}} E(z)=\frac{1}{t_{0}}\left(z-\overline{S_{\mathrm{i}}(z)}-\overline{S_{\mathrm{e}}(z)}\right)=\frac{1}{t_{0}}(z-\rho(z)) .
$$


Let us remark that this proof shows that the Schwarz function $S$ has, at least around infinity, the form

$$
S(z)=\sum_{k=1}^{n+1} k t_{k} z^{k-1}+\frac{t_{0}}{z}+\sum_{k=1}^{\infty} v_{k} z^{-k-1}
$$

where the $v_{k}=\frac{1}{\pi} \int_{D_{+}} z^{k} \mathrm{~d}^{2} z$ denote the harmonic moments of the interior domain $D_{+}$. This fact was already used in [2] to establish a connection between the harmonic moments and the coefficients of the parametrization of $\gamma$. And as was shown in [2], we find, with Theorems 4.1 and 6.1, that the $v_{k}$ are nothing but the expectation values of $\frac{t_{0}}{N} \operatorname{tr}\left(M^{k}\right)$ with respect to the probability measure $P_{N}(M) \mathrm{d} M$ in the limit $N \rightarrow \infty$ and hence, are completely determined by the harmonic moments of the exterior domain and $t_{0}$.

6.2. The Gaussian case. In this case, where the polynomial curve is an ellipse, we are able to calculate the equilibrium measure on $\mathbb{C}$ explicitly.

Proposition 6.4. The equilibrium measure $\mu$ for the potential

$$
V(z)=\frac{1}{t_{0}}\left(|z|^{2}-t_{2} z^{2}-\bar{t}_{2} \bar{z}^{2}\right), \quad\left|t_{2}\right|<\frac{1}{2},
$$

on $\mathbb{C}$ is

$$
\mu=\frac{1}{a b} \chi_{D_{+}} \lambda
$$

where $D_{+}$denotes the interior of the ellipse

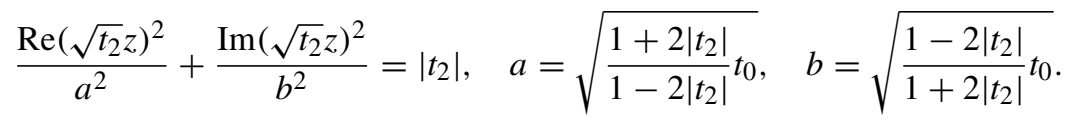

Proof. As a polynomial curve, the ellipse (13) has the parametrization

$$
h(w)=r\left(w+2 t_{2} w^{-1}\right), \quad r=\frac{a+b}{2} .
$$

We check that the given measure $\mu$ is the equilibrium measure by verifying the conditions of Corollary 3.5. To this end, let us introduce for $|w|>1$ the function

$$
\mathcal{E}(w)=E(h(w)), \quad E(z)=V(z)+\frac{2}{\pi t_{0}} \int_{D_{+}} \log \left|\frac{z}{\zeta}-1\right|^{-1} \mathrm{~d}^{2} \zeta .
$$

Integrating Eq. (12) and its complex conjugate analog we get for $\mathcal{E}(w)$ the expression

$$
\mathcal{E}(w)=\frac{1}{t_{0}}\left(|h(w)|^{2}-|h(1)|^{2}-2 \operatorname{Re} \int_{1}^{w} \bar{h}\left(w^{-1}\right) h^{\prime}(w) \mathrm{d} w\right) .
$$


Substituting in this expression relation (14) for $h$, we obtain

$$
\begin{aligned}
t_{0} \mathcal{E}(w)= & r^{2}\left(\left|w+2 t_{2} w^{-1}\right|^{2}-\left|1+2 t_{2}\right|^{2}\right) \\
& -2 r^{2} \operatorname{Re}\left(\bar{t}_{2} w^{2}+\left(1-4\left|t_{2}\right|^{2}\right) \log w+t_{2} w^{-2}-\left(t_{2}+\bar{t}_{2}\right)\right) \\
= & r^{2}\left(|w|^{2}-1-4\left|t_{2}\right|^{2}+4\left|t_{2}\right|^{2}|w|^{-2}+\left(1-4\left|t_{2}\right|^{2}\right) \log \left(|w|^{-2}\right)\right) \\
& +2 r^{2} \operatorname{Re}\left(2 t_{2} \bar{w} w^{-1}-t_{2}\left(\bar{w} w^{-1}\right)|w|^{-2}-t_{2}\left(\bar{w} w^{-1}\right)|w|^{2}\right) \\
= & r^{2}\left(|w|^{2}-1\right)\left(1-4\left|t_{2}\right|^{2}|w|^{-2}\right)+r^{2}\left(1-4\left|t_{2}\right|^{2}\right) \log \left(|w|^{-2}\right) \\
& -2 r^{2}\left(|w|^{2}-1\right)\left(1-|w|^{-2}\right) \operatorname{Re}\left(t_{2} \bar{w} w^{-1}\right) \\
= & r^{2}\left(|w|^{2}-1\right)\left(1-2\left|t_{2}\right|\right)\left(1+2\left|t_{2}\right||w|^{-2}\right)+r^{2}\left(1-4\left|t_{2}\right|^{2}\right) \log \left(|w|^{-2}\right) \\
& +2 r^{2}\left(|w|^{2}-1\right)\left(1-|w|^{-2}\right)\left(\left|t_{2}\right|-\operatorname{Re}\left(t_{2} \bar{w} w^{-1}\right)\right) .
\end{aligned}
$$

We are now ready to start estimating $\mathcal{E}(w)$ for $|w|>1$ and $\left|t_{2}\right|<\frac{1}{2}$. The last bracket we can estimate by

$$
\left|t_{2}\right|-\operatorname{Re}\left(t_{2} \bar{w} w^{-1}\right) \geq\left|t_{2}\right|-\left|t_{2} \bar{w} w^{-1}\right|=0 .
$$

It therefore remains to show

$$
\left(|w|^{2}-1\right)\left(1+2\left|t_{2}\right||w|^{-2}\right)+\left(1+2\left|t_{2}\right|^{2}\right) \log \left(|w|^{-2}\right) \geq 0,
$$

which follows immediately out of the following lemma.

Lemma 6.5. For $0 \leq \alpha \leq 1$ the function

$$
f(x)=(x-1)\left(1+\alpha x^{-1}\right)-(1+\alpha) \log x
$$

is non-negative on the interval $[1, \infty)$.

Proof. For the function $f$ and its derivatives $f^{\prime}$ and $f^{\prime \prime}$ we have

$$
f(1)=0, \quad f^{\prime}(1)=0 \quad \text { and } \quad f^{\prime \prime}(x)=\frac{1}{x^{2}}\left(1+\alpha-\frac{2 \alpha}{x}\right) \geq 0 .
$$

So we showed that $E(z) \geq 0$ for all $z \in \mathbb{C} \backslash D_{+}$. Because of Lemma 6.3, we also know that in the interior domain $D_{+}, E$ is zero and we therefore can apply Corollary 3.5 to see that $\mu$ is indeed the equilibrium measure for the potential $V$ on $\mathbb{C}$.

6.3. The proof of Theorem 6.1. As in the Gaussian case we are going to show that the measure $\mu$ given in the theorem fulfills the conditions of Corollary 3.5 and is therefore the uniquely defined equilibrium measure.

Because Theorem 6.1 is only valid for interior domains with small area, we are going to consider the asymptotical behaviour $t_{0} \rightarrow 0$, where the harmonic moments $\left(t_{k}\right)_{k=1}^{\infty}$ are kept fixed. To catch the asymptotical behavior of the corresponding polynomial curve, let us parametrize it as in the proof of Theorem 5.3:

$$
h(w)=r w+\sum_{j=0}^{n} r^{j} \alpha_{j} w^{-j}
$$

Then, for $r \rightarrow 0$, we have $r^{2} \simeq t_{0}, \alpha_{j} \simeq(j+1) \bar{t}_{j+1}, j \geq 1$, and, because we set $t_{1}=0$, $\alpha_{0} \simeq r^{2}$. 
Lemma 6.6. The critical radius of $h$ is asymptotically constant for $r \rightarrow 0$ :

$$
R=\sqrt{\left|\alpha_{1}\right|}+\mathcal{O}(r) .
$$

Proof. The roots of the function $h^{\prime}(w)=r-r \alpha_{1} w^{-2}-\cdots-n r^{n} \alpha_{n} w^{-n-1}$ are in zeroth order at $\pm \sqrt{\alpha_{1}}$ and ( $n-1$-times degenerated) at zero.

We consider now for $z \in D$ respectively for $w \in h^{-1}\left(D \backslash D_{+}\right)$the functions

$$
\begin{aligned}
& E(z)=V(z)+\int_{D_{+}} \log \left|\frac{z}{\zeta}-1\right|^{-1} \mathrm{~d}^{2} \zeta \text { and } \\
& \mathcal{E}(w)=E(h(w))=\frac{1}{t_{0}}\left(|h(w)|^{2}-|h(1)|^{2}+2 \operatorname{Re} \int_{1}^{w} \bar{h}\left(\tilde{w}^{-1}\right) h^{\prime}(\tilde{w}) \mathrm{d} \tilde{w}\right) .
\end{aligned}
$$

We already showed in Lemma 6.3 that $E(z) \equiv 0$ in $D_{+}$, so the first condition of the corollary is satisfied (this is essentially the way we have chosen our potential $V$ ).

Now, also with Lemma 6.3, we see that $E \geq 0$ in the vicinity of the curve $\gamma$, strictly speaking in the domain $h\left(B_{1 / R} \backslash \bar{B}_{1}\right)$. Indeed, if we look at the connected components of the contour lines of the function $E$ (which are smooth curves in the considered domain because there $\partial_{\bar{z}} E \neq 0$ ), we see that the gradient vector $\partial_{\bar{z}} E$ always points outwards, i.e. in the exterior domain of the contour line. Therefore, the value of $E$ on the contour lines is increasing outwards as desired.

A bit farther from the curve, i.e. for $1 / R \leq|w|<r^{-\alpha}, 0<\alpha<\frac{1}{3}$, the function $\mathcal{E}$ equals asymptotically the one of the corresponding ellipse $h^{(0)}(w)=r w+\alpha_{0}+$ $r \alpha_{1} w^{-1}$, we denote it by $\mathcal{E}^{(0)}$. Indeed, remarking that for the area of this ellipse we have $t_{0}^{(0)}=t_{0}+\mathcal{O}\left(r^{4}\right)$ and that $\mathcal{E}^{(0)}(w)=\mathcal{O}\left(r^{-2 \alpha}\right)$, we obtain, uniformly in $w$,

$$
\begin{aligned}
\mathcal{E}(w)-\mathcal{E}^{(0)}(w)= & \frac{1}{t_{0}}\left(|h(w)|^{2}-\left|h^{(0)}(w)\right|^{2}-|h(1)|^{2}+\left|h^{(0)}(1)\right|^{2}\right) \\
& +\frac{2}{t_{0}} \operatorname{Re} \int_{1}^{w}\left(h\left(\tilde{w}^{-1}\right)-h^{(0)}\left(\tilde{w}^{-1}\right)\right) h^{\prime}(\tilde{w}) \mathrm{d} \tilde{w} \\
& +\frac{2}{t_{0}} \operatorname{Re} \int_{1}^{w}\left(h^{\prime}(\tilde{w})-h^{(0)^{\prime}}(\tilde{w})\right) h^{(0)}\left(\tilde{w}^{-1}\right) \mathrm{d} \tilde{w} \\
& +\frac{1}{t_{0}}\left(t_{0}^{(0)}-t_{0}\right) \mathcal{E}^{(0)}(w) \\
= & \mathcal{O}\left(r^{1-\alpha}\right)+\mathcal{O}\left(r^{1-3 \alpha}\right)+\mathcal{O}\left(r^{1-2 \alpha}\right)+\mathcal{O}\left(r^{2-2 \alpha}\right) \\
\rightarrow & 0 \quad(r \rightarrow 0) .
\end{aligned}
$$

Because $\mathcal{E}^{(0)}(w) \geq C>0$ for all $|w| \geq \frac{1}{R}$ and $r>0$, we may choose $r$ so small that $\left|\mathcal{E}(w)-\mathcal{E}^{(0)}(w)\right|<\mathcal{E}^{(0)}(w)$ and so $\mathcal{E}(w)>0$ for all $w \in B_{r^{-\alpha}} \backslash B_{1 / R}$.

The domain remains, where $|w| \geq r^{-\alpha}$. For $k \geq 2$, we obtain

$$
\begin{aligned}
h(w)^{k}-(r w)^{k} & =\sum_{l=1}^{k}\left(\begin{array}{l}
k \\
l
\end{array}\right)(r w)^{k-l}\left(\sum_{j=0}^{n} r^{j} \alpha_{j} w^{-j}\right)^{l} \\
& =\sum_{l=1}^{k}\left(\begin{array}{l}
k \\
l
\end{array}\right) r^{k} w^{k-2 l}\left(\sum_{j=0}^{n} r^{j-1} \alpha_{j} w^{1-j}\right)^{l}=\mathcal{O}\left(r^{2}\right) .
\end{aligned}
$$


And since

$$
\begin{aligned}
t_{0} V(z) & =|z|^{2}-t_{2} z^{2}-\bar{t}_{2} \bar{z}^{2}+o\left(|z|^{2}\right) \\
& =\frac{1}{2}\left(\left|z-2 t_{2} \bar{z}\right|^{2}+\left(1-4\left|t_{2}\right|^{2}\right)|z|^{2}\right)+o\left(|z|^{2}\right)
\end{aligned}
$$

for $z \rightarrow 0$ and $V>0$ on $D \backslash\{0\}$, we may find a constant $C>0$ such that $t_{0} V(z) \geq C|z|^{2}$ for all $z$ in the compact domain $D$.

Therefore, for $r \rightarrow 0$,

$$
V(h(w))=V(r w)+\mathcal{O}(1) \geq \frac{C}{t_{0}}|r w|^{2}+\mathcal{O}(1),
$$

which tends to infinity at least as $r^{-2 \alpha}$.

On the other side, the integral over the logarithm in (15) diverges for $r \rightarrow 0$ only as $\log r$. So we have for $r$ small enough $E(h(w))>0$ for all $w \in h^{-1}\left(D \backslash D_{+}\right) \backslash B_{r^{-\alpha}}$.

This proves now $E(z) \geq 0$ for all $z \in D$ and therefore, with Corollary 3.5, that $\mu$ is the equilibrium measure.

6.4. Shifting the origin. As a little generalization, we consider the case where $t_{1} \neq 0$. This corresponds to a shift of the origin. Therefore, we like to define the harmonic moments also for a curve which does not encircle the origin.

Definition. Let

$$
h(w)=r w+\sum_{j=0}^{n} a_{j} w^{-j}
$$

parametrize a polynomial curve of degree $n$. Then the harmonic moments $\left(t_{k}\right)_{k=1}^{n+1}$ are given by the equation system (9). All other harmonic moments are set to zero.

Proposition 5.2 tells us that this definition coincides with the previous one if the origin is in the interior domain of the curve.

Corollary 6.7. For any set $\left(t_{k}\right)_{k=1}^{\infty} \subset \mathbb{C}$ with $\left|t_{2}\right|<\frac{1}{2}$ and $t_{k}=0$ for $k>n+1$ and any compact domain $D \subset \mathbb{C}$ such that $U(z)=|z|^{2}-2 \operatorname{Re} \sum_{k=1}^{n+1} t_{k} z^{k}$ has exactly one absolute minimum in the interior of $D$, there exists $a \delta>0$ so that for all $0<t_{0}<\delta$ the equilibrium measure $\mu$ for $V=\frac{1}{t_{0}} U$ on $D$ is given by

$$
\mu=\frac{1}{\pi t_{0}} \chi_{D_{+}} \lambda
$$

where $D_{+}$denotes the interior domain of the polynomial curve $\gamma$ defined by the harmonic moments $\left(t_{k}\right)_{k=0}^{\infty}$.

Proof. Let us first shift the origin by $a_{0}$, such that $V\left(z+a_{0}\right)$ has its absolute minimum in 0 . Thereby, the potential gets the form

$$
V\left(z+a_{0}\right)=\frac{1}{t_{0}}\left(|z|^{2}-2 \operatorname{Re} \sum_{k=2}^{n+1} t_{k}^{\prime} z^{k}\right)+V\left(a_{0}\right),
$$

where the $t_{k}^{\prime}$ are the harmonic moments of the shifted curve $\gamma-a_{0}$. Indeed, the coefficients of $V$ and the harmonic moments depend polynomially on the shift $a_{0}$. Because we know them to coincide as long as the origin is inside $D_{+}$, they do so for all $a_{0}$.

Applying now Theorem 6.1 for the shifted potential gives the desired result. 


\section{Discussion}

We have shown that under suitable assumptions on the polynomial $p$ appearing in the potential and on the integration range $D$ of the eigenvalues, the asymptotic density is uniform with support on a domain uniquely determined by the coefficients of the polynomial $p$.

It would be interesting to understand what happens at the range of validity of our assumptions. If the potential $V$ has more than one minimum in $D$ then one should expect for small $t_{0}$ to have an equilibrium measure with disconnected support, so that a description by a polynomial curve cannot be valid. Also as $t_{0}$ gets bigger, polynomial curves develop singularities and become non-simple. The question is then what happens to the eigenvalues. Finally we note that polynomial curves are (real sections of complex) rational curves. Curves of higher genus should arise by replacing $p$ by more general holomorphic functions.

\section{References}

1. Wiegmann, P. B., Zabrodin, A.: Conformal Maps and integrable hierarchies. Commun. Math. Phys. 213(3), 523-538 (2000)

2. Kostov, I. K., Krichever, I., Mineev-Weinstein, M., Wiegmann, P. B., Zabrodin, A.: $\tau$-function for analytic curves. In: Random matrix models and their applications, Math. Sci. Res. Inst. Publ. 40, Cambridge: Cambridge Univ. Press, 2001, pp. 285-299

3. Marshakov, A., Wiegmann, P., Zabrodin, A.: Integrable Structure of the Dirichlet Boundary Problem in Two Dimensions. Commun. Math. Phys. 227(1), 131-153 (2002)

4. Krichever, I., Marshakov, A., Zabrodin, A.: Integrable Structure of the Dirichlet Boundary Problem in Multiply-Connected Domains. http://arxiv.org/list/hep-th/0309010, 2003

5. Takasaki, K., Takebe, T.: Integrable Hierarchies and Dispersionless Limit. Rev. Math. Phys. 7(5), 743-808 (1995)

6. Chau, L.-L., Zaboronsky, O.: On the Structure of Correlation Functions in the Normal Matrix Model. Commun. Math. Phys. 196(1), 203-247 (1998)

7. Saff, E. B., Totik, V.: Logarithmic Potentials with External Fields. Grundlehren der mathematischen Wissenschaften 316, Berlin-Heidelberg-New York: Springer, 1997

8. Johansson, K.: On Fluctuations of Eigenvalues of Random Hermitian Matrices. Duke Math J. 91, 151-204 (1998)

9. Deift, P. A.: Orthogonal polynomials and random matrices: a Riemann-Hilbert Approach. Courant Lecture Notes in Mathematics, Vol 3, New York: Courant Institute of Mathematical Sciences, 1999

10. Davis, P. J.: The Schwarz function and its applications. The Carus Mathematical Monographs, No. 17, Washington, DC: The Mathematical Association of America, 1974 